\title{
Study of Ferroelectric and Dielectric Properties of Triglycine Selenate Crystal
}

\author{
ARVIND KUMAR RAWAT ${ }^{*}$, AANCHAL RAWAT and \\ TRILOK CHANDRA UPADHYAY
}

Physics Department, H.N.B. Garhwal University, Srinagar (Garhwal)- 246174, India

aanchalrawat@gmail.com; arvindsgfi@gmail.com

Received 7 December 2015 / Accepted 23 January 2016

\begin{abstract}
By including third- and fourth- order phonon anharmonic interactions terms into twosublattice pseudospin-lattice coupled mode model, expressions for shift, width, soft mode frequency, dielectric constant and loss tangent have been evaluated for ferroelectric TGSe crystal. The method of retarded double- time thermal Green's function has been used for derivation. Model values of physical quantities are fitted in expressions for soft mode frequency, shift, width, dielectric constant and loss tangent. Temperature dependence of these quantities has been numerically calculated. Theoretical results were compared with experimental results of available literature.
\end{abstract}

Keywords: Green's function, Dielectric, Anharmonic, Pseudospin, Loss tangent

\section{Introduction}

Ferroelectric crystals have attracted world-wide attention of physicists and engineers due to their potential use in modern technology; these are used in memory devices, pyroelectric detectors, modulators, capacitors, electro-optic, piezoelectric and other devices. Triglycine selenate crystal is one of best pyroelectric materials.

Triglycine selenate $\left(\mathrm{NH}_{2} \mathrm{CH}_{2} \mathrm{COOH}\right)_{3} \mathrm{H}_{2} \mathrm{SeO}_{4}$ is isomorphic to triglycine sulphate crystal. It is ferroelectric below $22.5{ }^{\circ} \mathrm{C}$. It shows a second order phase transition. The crystalline lattice is monoclinic. Above $T_{c}$ crystal belongs to $\mathrm{P} 21 / \mathrm{m}$ space group. TGSe is ferroelectric in the direction of a monoclinic axis $b$. The lattice parameters of TGSe are $a=9.520 \AA, b=12.847 \AA$, and $c=5.865 \AA$ and $\beta=110.5540$.

Experimental studies on TGSe crystal were made by a considerable number of workers. Pyroelectric properties of TGSe crystals were studied experimentally by Dziedzic and Mroz ${ }^{1}$. Bohdan et al., ${ }^{2}$ have done ellipsometric study of electronic excitations in TGSe crystals. They have measured real and imaginary parts of dielectric susceptibility of TGSe crystals in 4-9.8eV range. Lijewski et al., ${ }^{3}$ have done study of effect of uniaxial pressure on the ferroelectric 
phase transition in TGSe crystals. Castillo et al., ${ }^{5}$ have made Brillouin scattering studies of TGSe i.e. sound velocity versus uniaxial pressure. Romero et al., ${ }^{6}$ have carried out calorimetric and dielectric measurements on TGSe crystals. They have studied deuteration effects. Vorobyev et al., ${ }^{7}$ have done relaxation processes studies on TGSe crystal. They have measured real and imaginary parts of dielectric constant at different frequencies in TGSe crystal. Pandian et al., ${ }^{8}$ have done crystal growth study of TGSe crystals. Balasubramanyam et al., ${ }^{9}$ have done crystallization of TGSe type crystals in CdS nanoparticles. Khanum and Poder ${ }^{10}$ have done crystallization study of TGSe crystal doped with $\mathrm{NiSO}_{4}$. Batra et al., ${ }^{11}$ have done pyroelectric study of TGSe crystal experimentally. Renugadevi et al., ${ }^{12}$ have done crystal growth and characterization study of TGSe crystal. Gusowaska et al., ${ }^{13}$ have done terahertz and Raman Spectroscopy of TGSe Crystal and Chaudhury and Chitra et al. ${ }^{14}$ have done comparison between crystal structure parameters of triglycine Selenate and triglycine Sulphate crystal obtained from diffraction studies.

The theoretical studies have been made by Choudhury et al., ${ }^{15}$ by using two sublattice pseudospin lattice coupled mode model along with phonon anharmonic interactions terms. However, due to early decoupling of correlation functions at an early stage, they could not produce better and convincing results to explain ferroelectric transition and dielectric properties of TGSe crystal.

In the present work, we shall consider two-sublattice pseudospin-lattice coupled mode model along with third and fourth order phonon anharmonic interactions terms with the help of double-time thermal Green's function method, expressions for shift, width, soft mode frequency, dielectric constant and loss tangent have been derived. By fitting model values of physical parameters for TGSe crystal in the expressions for above quantities, their temperature dependence will be calculated numerically. The theoretically calculated results will be compared with experimental results of others ${ }^{16,17}$.

\section{Theory}

For the TGSe crystal, the ordering of the hydrogen bonds associated with glycine I and glycine II (or glycine III) hydrogen bonds should be taken into consideration. The pseudospin variables $S_{\alpha}$ are associated with ordering of the gylcine molecules ( $\alpha=$ I and II).

The two sublattice pseudospin model was suggested by Mitsui (originally for Rochelle salt crystal). We shall add into it third- order and fourth- order phonon anharmonic interactions terms. Thus, the Hamiltonian is expressed as;

$$
\begin{gathered}
H_{p}=-2 \Omega \sum_{i}\left(S_{1 i}^{x}+S_{2 i}^{x}\right)-\sum_{i j}\left[J_{i j}\left(S_{1 i}^{z} S_{1 j}^{z}+S_{2 i}^{z} S_{2 j}^{z}\right)+K_{i j} S_{1 i}^{z} S_{2 j}^{z}\right]-\sum_{k} V_{i k}\left(S_{1 i}^{z} A_{k}+S_{2 j}^{z} A_{k}^{+}\right)+ \\
\quad \frac{1}{4} \sum_{k} \omega_{k}\left(A_{k}^{+} A_{k}+B_{k}^{+} B_{k}\right) \\
+\sum_{\mathrm{k}_{1} \mathrm{k}_{2} \mathrm{k}_{3}} \mathrm{~V}^{3}\left(\mathrm{k}_{1}, \mathrm{k}_{2}, \mathrm{k}_{3}\right) \mathrm{A}_{\mathrm{k}_{1}} \mathrm{~A}_{\mathrm{k}_{2}} \mathrm{~A}_{\mathrm{k}_{3}}+\sum_{\mathrm{k}_{1} \mathrm{k}_{2} \mathrm{k}_{3} \mathrm{k}_{4}} \mathrm{~V}^{4}\left(\mathrm{k}_{1}, \mathrm{k}_{2}, \mathrm{k}_{3}, \mathrm{k}_{4}\right) \mathrm{A}_{\mathrm{k}_{1}} \mathrm{~A}_{\mathrm{k}_{2}} \mathrm{~A}_{\mathrm{k}_{3}} \mathrm{~A}_{\mathrm{k}_{4}}
\end{gathered}
$$

Where $S_{\alpha}^{m}$ (m=x,y or $\left.\mathrm{z}\right)$ is the $\mathrm{m}^{\text {th }}$ component of the pseudospin variable $S_{\alpha}, \Omega$ is proton tunnelling frequency, $\mathrm{J}_{\mathrm{ij}}$ and $\mathrm{K}_{\mathrm{ij}}$ are respectively, the coupling within the same group (I or II) and different groups. 
We shall consider the Green's function

$$
\mathrm{G}_{\mathrm{ij}}\left(\mathrm{t}-\mathrm{t}^{\prime}\right)=-\mathrm{i} \theta\left(\mathrm{t}-\mathrm{t}^{\prime}\right)<\left[S_{i}^{Z}(t) ; S_{j}^{Z}\left(t^{\prime}\right)\right]>=<<S_{1 i}^{Z}(t) ; S_{1 j}^{Z}\left(t^{\prime}\right)>>
$$

Now differentiating Green's function (2) with respect to $t$ and $t$ two times each, then fourier transforming and putting into Dyson's equation form $\mathrm{G}(\omega)=\mathrm{G}^{0}(\omega)+\mathrm{G}^{0}(\omega) \tilde{P}(\omega) \mathrm{G}^{0}(\omega)$. Finally the Green's function (2) as;

$$
\begin{gathered}
G_{i j}(\omega)=\frac{\Omega\left\langle S_{1 i}^{x}\right\rangle \delta_{i j}}{\pi\left[\omega^{2}-\hat{\Omega}^{2}-2 i \Omega \Gamma(\omega)\right]} \\
\text { where } \hat{\hat{\Omega}}^{2}=\tilde{\Omega}^{2}+2 \Omega \Delta(\omega) \\
\text { with } \tilde{\Omega}^{2}=a^{2}+b^{2}-b c \\
a=2 J_{i j}<s_{1}^{z}>+K_{i j}<s_{2}^{z}> \\
b=2 \Omega \\
c=2 J_{i j}<s_{1}^{x}>+K_{i j}<s_{2}^{x}> \\
\Delta(\omega)=\frac{a^{4} \quad b^{2} c^{2}}{2 \Omega\left(\omega^{2}-\tilde{\Omega}^{2}\right)}+\frac{V_{i k}^{2} N_{K} a^{2}}{2 \Omega\left(\omega^{2}-\tilde{\Omega}^{2}\right)}+\frac{2 V_{i k}^{2}\left\langle S_{1 i}^{x}\right\rangle \omega_{k} \delta_{k-k^{\prime}}\left(\omega^{2}-\tilde{\Omega}_{k}^{2}\right)}{\left(\omega^{2}-\tilde{\tilde{\omega}}_{k}^{2}\right)+4 \omega_{k}^{2} \Gamma_{k}^{2}(\omega)}
\end{gathered}
$$

and

$$
\Gamma(\omega)=\frac{\pi a^{4}}{4 \Omega \tilde{\Omega}}[\delta(\omega-\tilde{\Omega})-\delta(\omega+\tilde{\Omega})]+\frac{\pi V_{i k}^{2} N_{k} a^{2}}{4 \Omega \tilde{\Omega}}[\delta(\omega-\tilde{\Omega})-\delta(\omega+\tilde{\Omega})]+\frac{2 V_{i k}^{2}\left\langle S_{1 i}^{x}\right\rangle \omega_{k} \delta_{k-k^{\prime}}\left(\omega^{2}-\tilde{\tilde{\omega}}_{k}^{2}\right)}{\left(\omega^{2}-\tilde{\tilde{\omega}}_{k}^{2}\right)+4 \omega_{k}^{2} \Gamma_{k}^{2}(\omega)}
$$

The $\Delta(\omega)$ and $\Gamma(\omega)$ are real and imaginary parts of response function $\tilde{P}(\omega)$, called shift and width respectively. From Eq. (4) we obtain

$$
\hat{\Omega}_{ \pm}{ }^{2}=\frac{1}{2}\left[\left(\tilde{\Omega}^{2}+\tilde{\omega}_{k}^{2}\right) \pm\left\{\left(\tilde{\tilde{\omega}}_{k}^{2}-\tilde{\widetilde{\Omega}}^{2}\right)^{2}+8 V_{i k}^{2}\left\langle S_{1 i}^{x}\right\rangle \Omega\right\}^{\frac{1}{2}}\right]
$$

The frequency $\hat{\Omega}$ is Cochran's soft mode frequency which becomes zero as T tends to $\mathrm{T}_{\mathrm{C}}$, phase transition results and TGSe crystal becomes paraelectric. From Eq. (11) we can derive expression for dielectric constant $\mathrm{E}$ as

$$
\begin{aligned}
& \varepsilon=1+4 \pi \chi=1+4 \pi\left\{-2 \pi N \mu^{2} G(\omega)\right\} \\
& \varepsilon=-\frac{8 \pi N \mu^{2}\left\langle S_{1}^{x}\right\rangle}{\pi\left[\omega^{2}-\hat{\Omega}^{2}-2 \Omega i \Gamma(\omega)\right]}, \text { since } \varepsilon>>1 .
\end{aligned}
$$

From Eq. (13) dielectric tangent loss can be calculated as

$$
\begin{gathered}
\tan \delta=\frac{\text { Im aginarye }}{\operatorname{Re} a l \varepsilon} \\
=-\frac{2 \Omega \Gamma(\omega)}{\left(\omega^{2}-\hat{\Omega}^{2}\right)}
\end{gathered}
$$


From expressions (11), (12) and (15) we calculate numerically, temperature dependence of $\hat{\Omega}, \mathcal{\varepsilon}$ and $\tan \delta$ for TGSe crystal. We take values $\Omega=0.5 \mathrm{~cm}^{-1}, \mathrm{~J}=320 \mathrm{~cm}^{-1}, \mathrm{~K}=160 \mathrm{~cm}^{-1}$, $\mathrm{T}_{\mathrm{C}}=295.5 \mathrm{~K}, \mathrm{C}=4727 \mathrm{~K}, \mathrm{~V}_{\mathrm{ik}}=3 \mathrm{~cm}^{-1}, \mathrm{~N}_{\mathrm{ik}}=0.5, \omega_{\mathrm{k}}=0.69 \mathrm{~cm}^{-1}$ from literature.

In this way we obtaine theoretical values of soft mode frequency $\hat{\Omega}$ dielectric constant $\varepsilon$ and tangent loss $(\tan \delta)$ for TGSe crystal at different temperatures. The results have been shown in Figures (1)-(3) respectively. The theoretical results have been compared with experimentally reported result of Stankowaska and Hoshino et $\mathrm{al}^{16}$.

\section{Results and Discusion}

In the present calculations, correlation functions have been decoupled at proper stage. Earlier authors ${ }^{15}$ have decoupled these at early stage. Therefore, some important interactions disappeared from their results. We have also considered third- order phonon anharmonic interactions terms in our calculations. If third-order phonon interactions terms, shift and width are neglected from our calculations, our results at once reduce to the results of earlier authors ${ }^{15}$.

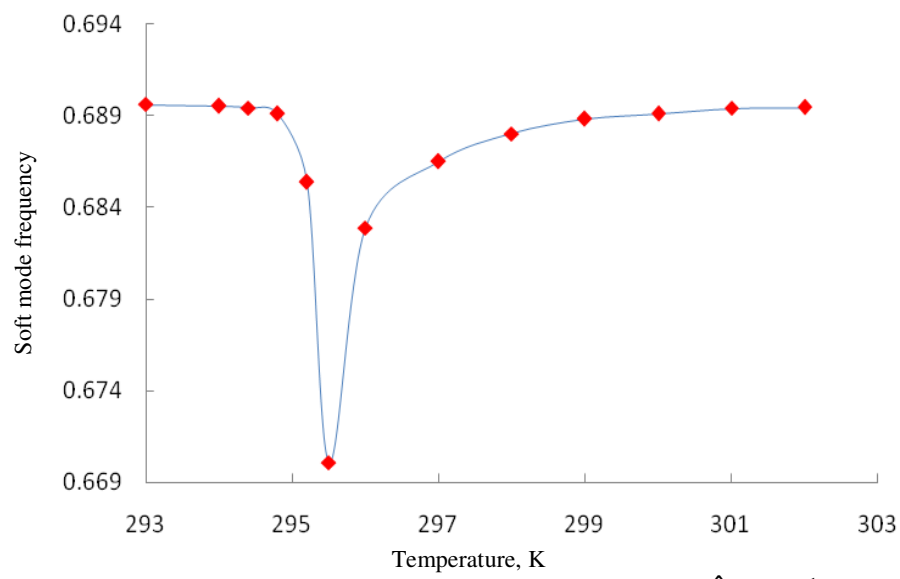

Figure 1. Temperature dependence of soft mode frequency $\hat{\Omega}\left(\mathrm{cm}^{-1}\right)$ of TGSe crystal (一 Our calculation; Experimentally correlated results of Stankowaska et al. ${ }^{17}$ )

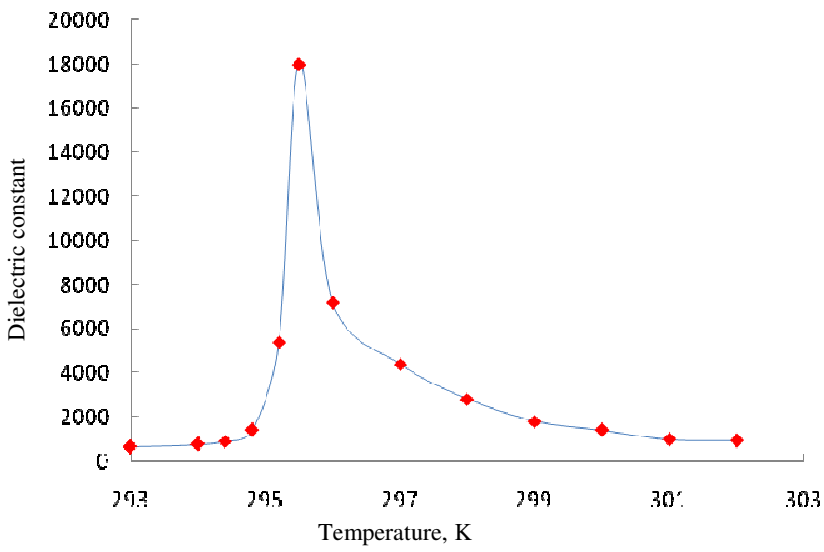

Figure 2. Temperature dependence of dielectric constant $(\epsilon)$ of TGSe crystal (- Our calculation; $\diamond$ Experimentally correlated results of Stankowaska et al.., ${ }^{17}$ ) 


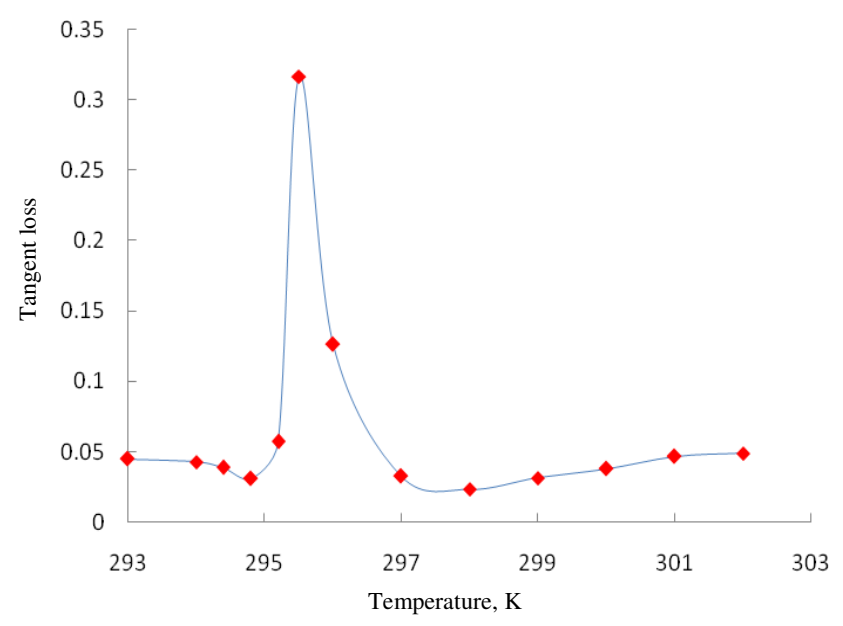

Figure 3. Temperature dependence of tangent loss $(\tan \delta)$ of TGSe

(— Our results; Experimentally correlated results of Stankowaska et al., ${ }^{17}$.)

The Eq.(11) along with Eqs.(6), (7) and (8) shows that soft mode frequency depends on tunnelling frequency $(\Omega)$, interchain $(\mathrm{J})$ and intrachain interactions $(\mathrm{K})$ respectively explictly. As temperature approaches $\mathrm{T}_{\mathrm{C}}$ soft mode frequency becomes zero. Our Eqs. (13) and (15) show that both $\epsilon$ and $\tan \delta$ depend on tunneling frequency, interchain and intrachain interactions as well as phonon anharmonic interactions respectively. Both increase anomalously in the vicinity of transition temperature. In the TGSe crystal isotope effect occurs both on $\mathrm{T}_{\mathrm{C}}$ and $\mathrm{C}$. Therefore, the two-sublattice model is suitable here. Our theoretical expressions predict the behaviour of TGSe crystal in agreement with experimental results. Our theoretical results shown in Figures 1, 2 and 3 show close agreements with experimental results of Hoshino et al., ${ }^{16}$ and Stankowaska et al. ${ }^{17}$.

\section{Conclusion}

From the present study, it emerges that using the two sublattice pseudospine-lattice coupled mode model along with third-and fourth-order phonon anharmonic terms and double-time thermal Green's function method, the temperature variations of dielectric constant and loss tangent in TGSe crystal gives more adequately and better temperature variations of dielectric constant and loss tangent in TGSe crystal. The crystals of TGSe are better materials for pyroelectric detection. By using expression obtained in the Present study may also be used to explain ferroelectric and dielectric properties of other similar crystals triglycine sulphate, triglycine fluoroberyllate, ammonium bisulphate successfully.

\section{Acknowledgement}

The Authors are grateful to Prof B S Semwal (Former Head, Physics Deptt) for his blessings and encouragements. They are thankful to Prof U C Naithani, Prof R P Gairola, Prof S C Bhatt, Dr Hemant Singh Parmar and Dr Manish Uniyal, Mrs Meera Rawat for their encouragements.

\section{References}

1. Dziedzic J and Mrog J, Acta Physica Polonica A, 2005, 108(6), 971-978; DOI:10.12693/AphysPolA.108.971 
2. Bohdan A, Patryn A, Cobet C, Mytsyk B and Esser N, Physica Status Solidi (b), 2009, 346(10), 2337-2340; DOI:10.1002/pssb.200844384

3. Ligewski S, Goslar J and Hoffmann S K, J Phys: Condensed Matter, 2006, 18(26), 6159-6169; DOI:10.1088/0953-8984/18/26/033.

4. Marcelli K, Stankowska J, Iglesias T and Julio A G, J Phys Condens Matter, 1996, 8(22), 4079-4093.

5. Casttillo J R, Gonzalo J A, Mroz B and Tylczynski Z, Physica B Condensed Matter, 1999, 262(3-4), 433-437; DOI:10.1016/S0921-4526(98)01091-6

6. Romero F J, Gallardo M C, Martin-Olalla J M and Cerro D J, J Appl Phys., 2010, 107, 124110.

7. Vorobyev A V, Gavrilova N D and Lotonov A M, Moscow University Physics Bulletin, 2014, 69(2), 175-179; DOI:10.3103/S0027134914020131

8. Pandian M S, Ramasami P and Kumar B, Mat Res Bull., 2012, 47, 1587-1597.

9. Balasubrahmanyam K, Kumar E and Selverajan P, Indian J Sci Techn., 2010, 3(1), 41; DOI:10.17485/ijst/2010/v3i1/29641

10. Khanum and Podder, J Cryst Proc Tech., 2001, 1, 49-54; DOI:10.4236/jcpt.2011.13008

11. Batra, A K, Bhattacharjee S, Chilvery A K, Aggarwal M D, Edwards M E and Bhalla A J, Photon Energy., 2011, 1(1), 014001-014012; DOI:10.1117/1.3656395

12. Renugadevi R, Kanchana J, Pandian M S, Ramaswmi P and Kumar B, Mate Res Bull (in Press). (2012).

13. Gusowska M T, Plinski E F, Baran J, Walczakowski M J, Jarzab P P, Nowak K, Fuglewicz B, Mikvlics M, Palka N and Szvstakowski M, Optical Mater., 2014, 37, 28-35; DOI:10.1016/j.optmat.2014.04.032

14. Choudhury R R and Chitra R, J Phys: Condensed Matter, 2009, 21(33), 335901; DOI:10.1088/0953-8984/21/33/335901

15. Choudhury K R, Nath D, Banerjee S and Chaudhuri B K, Phys Rev B, 1982, 26, 6276; DOI:10.1103/PhysRevB.26.6276

16. Hoshino S, Mitsui T, Jona F and Pepinsky R, Phys Rev, 1957, 107, 1255; DOI:10.1103/PhysRev.107.1255

17. Stankowaska R, Czarneeka A and Kaluba M, Acta Phys Polanica A, 1976, 50, 823. 